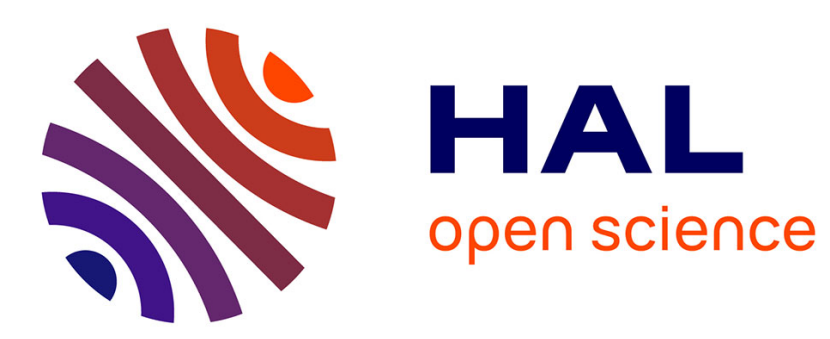

\title{
Three-dimensional Tracking with Angle Measurements without Observer Maneuver
}

Christian Musso, Patrick Fabiani

\section{To cite this version:}

Christian Musso, Patrick Fabiani. Three-dimensional Tracking with Angle Measurements without Observer Maneuver. FUSION 2018, Jul 2018, CAMBRIDGE, United Kingdom. 10.23919/ICIF.2018.8455814 . hal-02301723

\section{HAL Id: hal-02301723 \\ https://hal.science/hal-02301723}

Submitted on 30 Sep 2019

HAL is a multi-disciplinary open access archive for the deposit and dissemination of scientific research documents, whether they are published or not. The documents may come from teaching and research institutions in France or abroad, or from public or private research centers.
L'archive ouverte pluridisciplinaire HAL, est destinée au dépôt et à la diffusion de documents scientifiques de niveau recherche, publiés ou non, émanant des établissements d'enseignement et de recherche français ou étrangers, des laboratoires publics ou privés. 


\section{Three-dimensional Tracking with Angle Measurements without Observer Maneuver}

\author{
Christian Musso \\ ONERA - The French Aerospace Lab \\ F-91761 Palaiseau, France \\ Email: christian.musso@onera.fr
}

\author{
Patrick Fabiani \\ ISAE-SUPAERO \\ 31055 TOULOUSE, France \\ Email: patrick.fabiani@isae-supaero.fr
}

\begin{abstract}
Passive target estimation is a widely investigated problem of practical interest. We are concerned specifically with an autonomous flight system developed onboard the ONERA ReSSAC unmanned helicopter. This helicopter is equipped with a (visible or infrared) camera and so is able to measure azimuths and elevation angles of a target. The latter is supposed to follow a constant velocity motion. It is well known that observer must maneuver in order to insure the observability of the target state. We are interested in tracking partly the target state when both the observer and the target have a constant velocity model in a three-dimensional space. We describe the set of all the trajectories compatible with the angle measurements and we propose a quick method to estimate these trajectories.
\end{abstract}

\section{INTRODUCTION}

Passive target estimation is a widely investigated problem of practical interest. We are concerned specifically with an autonomous flight system developed onboard the ONERA ReSSAC unmanned helicopter [1]. This helicopter is equipped with a (visible or infrared) camera and so is able to perform lines of sight of the targets. More generally, we consider a single moving observer which measures the azimuths and the elevation angles of a moving target in a three-dimensional space. If all measurements are derived from a single observer and consist of angles only, it is well known that target state observability is warranted only under specific conditions on the observer trajectory. The observer must maneuver in order to insure the observability of the target state. Optimal maneuvers have been studied for the purpose of improving the accuracy of the target estimation for bearing-only tracking [2], [3], [4]. The aim of the paper is to describe and to estimate the set of all the trajectories (called ambiguous) compatible with the angle measurements in case were both the observer and the target follow a constant velocity motion. This kind of problem has been studied in bearings only tracking in a two-dimensional space like in [5]. In the three-dimensional context, the ambiguous trajectories can be determined by the observer as they depend of three azimuths and two elevation angles. We show how these angles can be quickly estimated. A possible application is to discriminate among the ambiguous trajectories in exploiting an a priori knowledge about the target state.

The paper is organized as follows. The general framework is presented in section II. Section III recall the conditions ensuring the observability of the target. In this section we calculate the set of all the target trajectories compatible with the angle measurements in the case where both the target and the observer follow a uniform motion. We present in section IV a way to estimate these ambiguous trajectories thanks to a linear estimator. Section V shows some simulation results and finally, some conclusions are drawn in section VI.

\section{PROBLEM STATEMENT}

It is assumed that the target follows a constant velocity model in a three-dimensional space. In a discrete time model, the target state is defined by

$$
X_{T}\left(t_{k}\right)=\left[x_{T}\left(t_{k}\right), \dot{x}_{T}, y_{T}\left(t_{k}\right), \dot{y}_{T}, z_{T}\left(t_{k}\right), \dot{z}_{T}\right]^{T}
$$

The target evolves as follows

$$
X_{T}\left(t_{k}\right)=F X_{T}\left(t_{k-1}\right)+\eta\left(t_{k}\right)
$$

where $\eta\left(t_{k}\right)$ is a white Gaussian noise process and where the state transition matrix is defined by

$$
F=\left[\begin{array}{cccccc}
1 & \Delta T & 0 & 0 & 0 & 0 \\
0 & 1 & 0 & 0 & 0 & 0 \\
0 & 0 & 1 & \Delta T & 0 & 0 \\
0 & 0 & 0 & 1 & 0 & 0 \\
0 & 0 & 0 & 0 & 1 & \Delta T \\
0 & 0 & 0 & 0 & 0 & 1
\end{array}\right]
$$

$\Delta T \triangleq t_{k}-t_{k-1}$ being the sampling period. The noise-free azimuths and elevation angles satisfy the nonlinear equation (Fig. 1)

$$
\left\{\begin{array}{l}
\theta(t)=\arctan \left[\frac{r_{y}(t)}{r_{x}(t)}\right] \\
\phi(t)=\arctan \left[\frac{r_{z}(t)}{\sqrt{r_{x}^{2}(t)+r_{y}^{2}(t)}}\right]
\end{array}\right.
$$

where

$$
\begin{aligned}
& r(t)=\left(r_{x}(t), r_{y}(t), r_{z}(t)\right)^{T}= \\
& \left(x_{T}(t)-x_{o}(t), y_{T}(t)-y_{o}(t), z_{T}(t)-z_{o}(t)\right)^{T}
\end{aligned}
$$

is the vector of the relative positions between the target and the observer. First we recall, in a simple way, the necessary conditions under which the target is observable. 


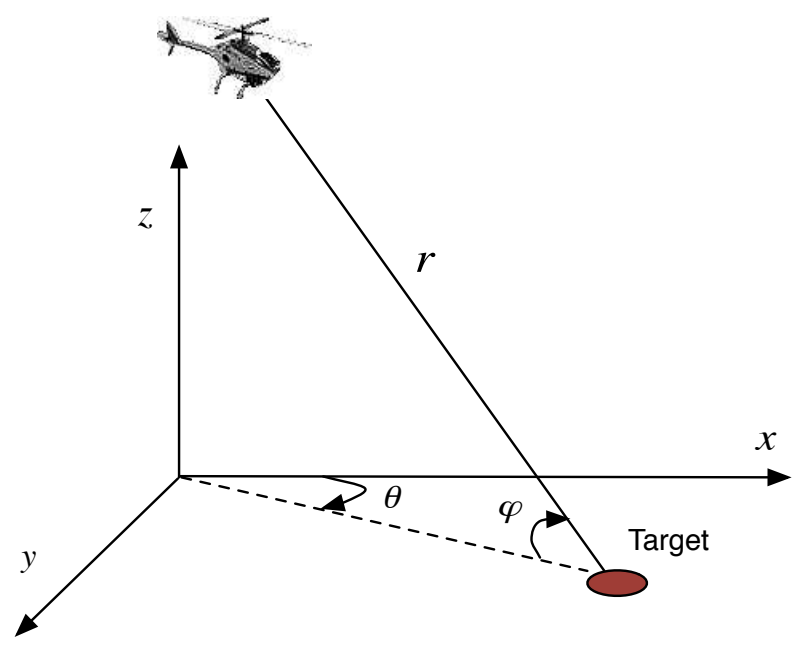

Fig. 1. Observer geometry. For better legibility the target is represented on the ground

\section{Ambiguous TRAJECTORIES}

\section{A. Observability conditions}

The observability conditions of three-dimensional estimations with azimuths and elevation angles have been widely studied [6], [7]. The authors analyze the observability (here in a nonlinear context) in a linear framework, thus allowing direct utilization of a simplified observability criterion. A direct application of the following proposition [7] states the necessary conditions under which the target state is observable.

Proposition 1. Let $r(t)=\left(r_{x}(t), r_{y}(t), r_{z}(t)\right)^{T}$ be the relative position vector (4). If the target state is observable then

$$
\left\{\begin{array}{l}
r_{x}(t) \neq \alpha(t) P_{1}(t) \\
r_{y}(t) \neq \alpha(t) P_{2}(t) \\
r_{z}(t) \neq \alpha(t) P_{3}(t) .
\end{array}\right.
$$

where $P_{1}(t), P_{2}(t), P_{3}(t)$ are any polynomials of degree the order of the target model dynamics and where $\alpha(t)$ is an arbitrary scalar function

The order of the dynamics should be understood as the Mthorder of the series expansion in time of the dynamics. For example $M=0$ corresponds to a stationary target, $M=1$ corresponds to a trajectory of constant target. In others words, the proposition states that the order of the observer dynamics must be different of the order of the target dynamics. On the contrary, if the target and observer have a constant velocity model,

$$
r(t)=r\left(t_{0}\right)+\left(t-t_{0}\right) \dot{r}\left(t_{0}\right)
$$

$r_{x}(t), r_{y}(t), r_{z}(t)$ are polynomials of degree one which is also the degree of the target dynamics. So the target state is not observable in this case. Any maneuver of the observer $(M \geq 2)$ enables generally observability. However, the conditions of the Proposition 1 are necessary but not sufficient. Indeed, for example, if the target is stationary and the observer follows a constant velocity model along the line of sight, the target is unobservable while the order of the observer dynamics is greater than the one of the target.

We are now interested in showing all the trajectories compatible with the angle measurements when both the observer and the target have a constant velocity model.

\section{B. Ambiguous trajectories}

If both the observer and the target have a constant velocity model (2), there exists different constant velocity trajectories of the target which match with the angle measurements. The next proposition establishes the set of all of such trajectories called ambiguous trajectories.

Let $X_{o}\left(t_{0}\right)$ be the initial 6-dimensional state vector of the observer. Let $t_{1}, t_{2}, t_{3}$ be three distinct times and $t_{1}^{\prime}, t_{2}^{\prime}$ be two distinct times. Let us introduce some notations

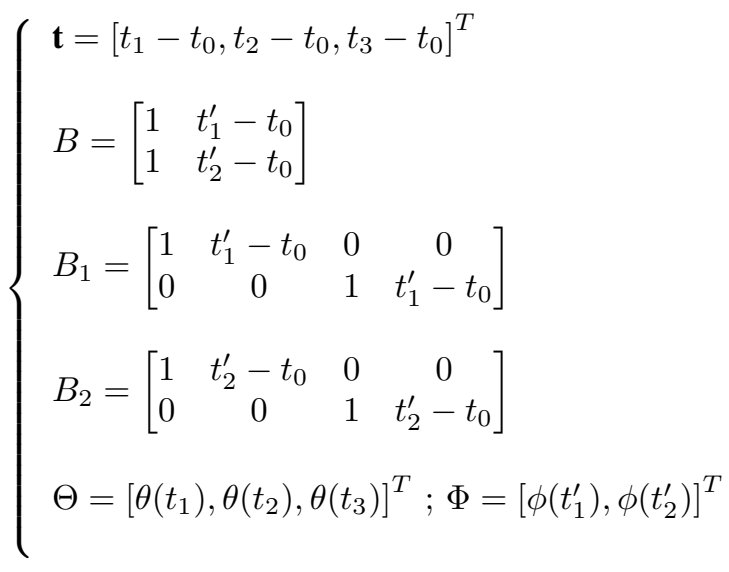

The vector $(\Theta, \Phi)$ consists in 3 azimuths and 2 elevation angles evaluated exactly at the times $\left(t_{1}, t_{2}, t_{3}\right)$ and $\left(t_{1}^{\prime}, t_{2}^{\prime}\right)$ respectively.

Proposition 2. Assume that both the observer and the target follow a constant velocity motion. The ambiguous target trajectories which are compatible with the azimuths and elevation angles $(\theta(t), \phi(t))(3)$ are determined by the following initial target state $X_{T}\left(t_{0}, \mu\right)(1)$

$$
X_{T}\left(t_{0}, \mu\right)=X_{o}\left(t_{0}\right)+\mu\left[\begin{array}{l}
A_{\Theta}^{-1} \boldsymbol{t} \\
1 \\
B^{-1} \boldsymbol{s}_{\Theta, \Phi}
\end{array}\right]
$$

where $\mu$ is an arbitrary non-zero scalar and where

$$
\begin{gathered}
A_{\Theta}=\left[\begin{array}{lll}
\tan \theta\left(t_{1}\right) & \left(t_{1}-t_{0}\right) \tan \theta\left(t_{1}\right) & -1 \\
\tan \theta\left(t_{2}\right) & \left(t_{2}-t_{0}\right) \tan \theta\left(t_{2}\right) & -1 \\
\tan \theta\left(t_{3}\right) & \left(t_{3}-t_{0}\right) \tan \theta\left(t_{3}\right) & -1
\end{array}\right] \\
\boldsymbol{s}_{\Theta, \Phi}=\left(\begin{array}{c}
\left\|B_{1} u_{\Theta}\right\| \tan \phi\left(t_{1}^{\prime}\right) \\
\left\|B_{2} u_{\Theta}\right\| \tan \phi\left(t_{2}^{\prime}\right)
\end{array}\right)
\end{gathered}
$$


with $u_{\Theta}=\left(\begin{array}{c}A_{\Theta}^{-1} \boldsymbol{t} \\ 1\end{array}\right)$. The ambiguous trajectories defined by (8) are determined by the 5 angles $(\Theta, \Phi)$ (7) and are set by a non-zero scalar $\mu$.

\section{PROOF OF PROPOSITION 2.}

An ambiguous trajectory in the three-dimensional space remains ambiguous in the $x y$ plane. Therefore we analyze first the $x y$ ambiguous trajectories only taking into account the azimuths. For $i=1,2,3$ we have

$$
\tan \theta\left(t_{i}\right)=\frac{r_{y}\left(t_{0}\right)+\left(t_{i}-t_{0}\right) r_{\dot{y}}\left(t_{0}\right)}{r_{x}\left(t_{0}\right)+\left(t_{i}-t_{0}\right) r_{\dot{x}}\left(t_{0}\right)}
$$

Let $Y=\left[r_{x}\left(t_{0}\right), r_{\dot{x}}\left(t_{0}\right), r_{y}\left(t_{0}\right)\right]^{T}$. Let us define by $\mu$ the parameter: $\mu=r_{\dot{y}}\left(t_{0}\right)$. The whole 4-dimensional state vector $[Y, \mu]^{T}$ in the $x y$ plane is characterized by the three azimuths. Indeed, equation (11) gives

$$
A_{\Theta} Y=\mu\left[\begin{array}{c}
t_{1}-t_{0} \\
t_{2}-t_{0} \\
t_{3}-t_{0}
\end{array}\right]=\mu \mathbf{t}
$$

where $A_{\Theta}$ is defined in (9) and $\mathbf{t}$ in (7). Therefore, the set of the ambiguous trajectories in the $x y$ plane is characterized by the initial relative following state vector

$$
\left[r_{x}\left(t_{0}\right), r_{\dot{x}}\left(t_{0}\right), r_{y}\left(t_{0}\right), r_{\dot{y}}\left(t_{0}\right)\right]^{T}=\left[\mu A_{\Theta}^{-1} \mathbf{t}, \mu\right]^{T}
$$

These 4-dimensional trajectories are the projections of the ambiguous trajectories in the 6-dimensional space. Thus, it remains now to determine the initial relative height $r_{z}\left(t_{0}\right)$ and the initial relative vertical velocity $r_{\dot{z}}\left(t_{0}\right)$ which are compatible with the exact elevation angles $\phi(t)$. It is sufficient to consider the two elevation angles $\phi\left(t_{1}^{\prime}\right)$ and $\phi\left(t_{2}^{\prime}\right)$ (7). We have for $i=1,2$

$$
\tan \phi\left(t_{i}^{\prime}\right)=\frac{r_{z}\left(t_{0}\right)+\left(t_{i}^{\prime}-t_{0}\right) r_{\dot{z}}\left(t_{0}\right)}{\left[r_{x}\left(t_{i}^{\prime}\right)^{2}+r_{y}\left(t_{i}^{\prime}\right)^{2}\right]^{1 / 2}}
$$

Using the definition of $B(7)$, the equation (14) yields

$\left[r_{z}\left(t_{0}\right), r_{\dot{z}}\left(t_{0}\right)\right]^{T}=B^{-1}\left[\begin{array}{c}\left(r_{x}\left(t_{1}^{\prime}\right)^{2}+r_{y}\left(t_{1}^{\prime}\right)^{2}\right)^{1 / 2} \tan \phi\left(t_{1}^{\prime}\right) \\ \left(r_{x}\left(t_{2}^{\prime}\right)^{2}+r_{y}\left(t_{2}^{\prime}\right)^{2}\right)^{1 / 2} \tan \phi\left(t_{2}^{\prime}\right)\end{array}\right]$

The relative positions $r_{x}\left(t_{1}^{\prime}\right), r_{y}\left(t_{1}^{\prime}\right), r_{x}\left(t_{2}^{\prime}\right)$ and $r_{y}\left(t_{2}^{\prime}\right)$ can be deduced from the initial relative state vector (13) in the $x y$ plane and from (6). After some calculations, we get

$$
\left[r_{z}\left(t_{0}\right), r_{\dot{z}}\left(t_{0}\right)\right]^{T}=\mu B^{-1} \mathbf{s}_{\Theta, \Phi}
$$

where $\mathbf{s}_{\Theta, \Phi}$ is defined in (10). Finally, by combining equations (13) and (15), we get the following family of 6-dimensional initial state vectors of the target $X_{T}\left(t_{0}, \mu\right)$

$$
X_{T}\left(t_{0}, \mu\right)=X_{o}\left(t_{0}\right)+\mu\left[\begin{array}{l}
A_{\Theta}^{-1} \mathbf{t} \\
1 \\
B^{-1} \mathbf{s}_{\Theta, \Phi}
\end{array}\right]
$$

For any $\mu \neq 0, X_{T}\left(t_{0}, \mu\right)$ generates an ambiguous trajectory which produces the set of azimuths and elevation angles $(\theta(t), \phi(t))$ characterized by $(\Theta, \Phi)(7)$. Note that in the case where $\mu=0$, the ambiguous trajectory match that of the observer trajectory: $X_{T}\left(t_{0}, 0\right)=X_{o}\left(t_{0}\right)$. The function tangent involved in (9) and (10) being of period $\pi$, it is sufficient to consider positive values of $\mu$. Negative values produce point symmetrical trajectories.

It remains now to estimate the vector $(\Theta, \Phi)$ when the angle measurements are noisy. This vector is of course always observable.

\section{ESTIMATION OF THE AMBIGUOUS TRAJECTORIES}

We present a quick method to estimate the ambiguous trajectories using only the angle measurements. The observer and the target motions still have a constant velocity model. For that purpose, we have to estimate the vector $(\Theta, \Phi)(7)$ which determine all the ambiguous trajectories by varying the parameter $\mu$ (see proposition 2). A useful method, based on the Gauss quadrature, allows us to estimate quickly and accurately these angles providing that the variation amplitude of the angles is not too large. The 5 angles $(\Theta, \Phi)$ are evaluated by means of a linear combination of the measurements.

\section{A. Description of the method}

Let $t_{1}<\cdots<t_{n}$ be $n$ positive reals. Let us denote by $l^{2}$ the set of real valued functions $f$ defined on $\mathbb{R}$ such that

$$
\sum_{t=t_{1}}^{t_{n}} f^{2}(t)<\infty
$$

$l^{2}$ is endowed with the inner product $\langle\cdot, \cdot\rangle$ defined by

$$
\langle f, g\rangle=\sum_{t=t_{1}}^{t_{n}} f(t) g(t)
$$

for any $f, g \in l^{2}$. For any $1 \leq m \leq n$, let us denote by $\Psi_{m}$, the Legendre Polynomial of degree $m$ such that

$$
\left\langle\Psi_{m}, t^{j}\right\rangle=0
$$

for any integer $0 \leq j \leq m-1$. $\Psi_{m}$ can be computed recursively using the Graham-Schmidt formula [8]. Let us denote by $T_{1} \leq \cdots \leq T_{m}$ the roots of $\Psi_{m}$. One can prove that for any $i \neq j$ with $1 \leq i, j \leq m, T_{i}$ is a real such that

$$
t_{1} \leq T_{i} \leq t_{n} \quad \text { and } \quad T_{i} \neq T_{j} .
$$

If $m=n$, then $T_{i}=t_{i}$, for any $1 \leq i \leq m=n$.

The roots of $\Psi_{m}$ can be computed approximately by the Laguerre algorithm [8]. 
For any $1 \leq i \leq m$, let us introduce $\Phi_{i}$ the Lagrange polynomial of degree $\mathrm{m}-1$ such that

$$
\Phi_{i}(t)= \begin{cases}1 \quad \text { if } \quad t=T_{i} \\ 0 \quad \text { if } \quad t=T_{j} \neq T_{i} .\end{cases}
$$

We consider now the following measurement model,

$$
z(t)=\beta(t)+\varepsilon(t)
$$

where $\varepsilon(t)$ are i.i.d. centered Gaussian noises with standard deviation (std) $\sigma . \beta(t)$ plays the role of the elevation angles or the role of the azimuths (3). Let $n$ be the number of observations. We aim to estimate $m$ values of $\beta(t)$ for $t=T_{1}, T_{2}, \ldots, T_{m}$ with $1<m<n$. The following proposition exhibits an estimator of the angles $\beta_{T_{1}}, \ldots, \beta_{T_{m}}$ by means of a linear combination of the measurements $z(t)$ (19).

Proposition 3. The following assertions holds.

1) For $i=1,2, \ldots m$, the estimators

$$
\hat{\beta}_{T_{i}}=\frac{1}{\left\|\Phi_{i}\right\|^{2}} \sum_{t=t_{1}}^{t_{n}} \Phi_{i}(t) z(t)
$$

are nearly unbiased

$$
\mathbb{E}\left(\hat{\beta}_{T_{i}}\right) \approx \beta_{T_{i}}
$$

where $\Phi_{i}$ is the Lagrange polynomial of degree $m-1$ defined in (18).

2) The covariance matrix of the estimators is diagonal. For $i, j=1,2, \ldots m$, we have

$$
\begin{gathered}
\operatorname{cov}\left(\hat{\beta}_{T_{i}}, \hat{\beta}_{T_{j}}\right)=\delta_{i=j} \frac{\sigma^{2}}{\left\|\Phi_{i}\right\|^{2}} \\
\text { with }\left\|\Phi_{i}\right\|^{2}=\sum_{t=t_{1}}^{t_{n}} \Phi_{i}^{2}(t)
\end{gathered}
$$

These suitable properties (21) and (22) are obtained using the orthogonality of the Lagrange polynomials (18) which interpolate the roots of the Legendre polynomial [9], [10], [11]. One can prove that $\left\|\Phi_{i}\right\|^{2} \sim$ constant $\times n$, so that the std of $\hat{\beta}_{T_{i}}$ is of order $\frac{\sigma}{\sqrt{n}}$ when $n$ is large.

The linear estimator (20) is very accurate providing that the signal has a dynamics order less then $2 m$, namely, the signal must behave as a polynomial with a degree lower or equal to $2 m-1$.

\section{B. Application to the estimation of the ambiguous trajectories}

We consider we have $n$ noisy azimuths and elevation angles (3) measurements according to the model

$$
\left\{\begin{array}{c}
z_{\theta}(t)=\theta(t)+\varepsilon(t) \\
z_{\phi}(t)=\phi(t)+\nu(t)
\end{array}\right.
$$

where $\varepsilon(t)$ and $\nu(t)$ are i.i.d. centered Gaussian noises with std $\sigma_{\theta}$ anf $\sigma_{\phi}$ respectively. $\theta(t)$ and $\phi(t)$ are defined in (3). Here, we do not take into account the relation between these angles and the whole state $X_{T}(t)(1)$. We aim to estimate the azimuths and elevation angles at times $\left(t_{1}, t_{2}, t_{3}\right)$ and $\left(t_{1}^{\prime}, t_{2}^{\prime}\right)$ respectively using only the angle measurements (23). For this purpose we choose $\left(t_{1}, t_{2}, t_{3}\right)=\left(T_{1}, T_{2}, T_{3}\right)$ and $\left(t_{1}^{\prime}, t_{2}^{\prime}\right)=\left(T_{1}^{\prime}, T_{2}^{\prime}\right)$ where $\left(T_{1}, T_{2}, T_{3}\right)$ are the roots of the Legendre polynomial $\Psi_{m}$ (17) with $m=3$ and where $\left(T_{1}^{\prime}, T_{2}^{\prime}\right)$ are the roots of the Legendre polynomial $\Psi_{m}$ with $m=2$.

Consequently, the estimations of $(\Theta, \Phi)$ (7) are done using the linear estimator (20).

\section{Estimation of $(\Theta, \Phi)$}

1) For $i=1,2,3$

$$
\hat{\theta}_{T_{i}}=\frac{1}{\left\|\Phi_{i}\right\|^{2}} \sum_{t=t_{1}}^{t_{n}} \Phi_{i}(t) z_{\theta}(t)
$$

2) For $i=1,2$

$$
\hat{\phi}_{T_{i}^{\prime}}=\frac{1}{\left\|\Phi_{i}^{\prime}\right\|^{2}} \sum_{t=t_{1}}^{t_{n}} \Phi_{i}^{\prime}(t) z_{\phi}(t)
$$

The Lagrange polynomials $\Phi_{i}$ and $\Phi_{i}^{\prime}$ (18) interpolate $\left(T_{1}, T_{2}, T_{3}\right)$ and $\left(T_{1}^{\prime}, T_{2}^{\prime}\right)$ respectively.

In the case of the Legendre polynomial with degree $m=3$ or $m=2$ and when $t_{k}=k$ for $1 \leq k \leq n$, the roots can be computed exactly with the following formula.

\section{Roots of the Legendre polynomials}

1) Case $m=3$

$$
\begin{aligned}
& T_{1}=\frac{n+1}{2}-\tau, \quad T_{2}=\frac{n+1}{2}, \quad T_{3}=\frac{n+1}{2}+\tau, \\
& \left\|\Phi_{1}\right\|^{2}=\left\|\Phi_{3}\right\|^{2}=\frac{5 n\left(n^{2}-1\right)}{6\left(3 n^{2}-7\right)}, \quad\left\|\Phi_{2}\right\|^{2}=\frac{4 n\left(n^{2}-4\right)}{3 n^{2}-7} \\
& \text { with } \tau=\sqrt{\frac{3 n^{2}-7}{20}}
\end{aligned}
$$

2) Case $m=2$

$$
\begin{aligned}
& \quad T_{1}^{\prime}=\frac{n+1}{2}-\tau, \quad T_{2}^{\prime}=\frac{n+1}{2}+\tau, \\
& \left\|\Phi_{1}^{\prime}\right\|^{2}=\left\|\Phi_{2}^{\prime}\right\|^{2}=\frac{n}{2}, \\
& \text { with } \tau=\sqrt{\frac{n^{2}-1}{12}}
\end{aligned}
$$

The estimators (24) (25) are nearly unbiased providing that the variation amplitude of the angles is not too large. For $i=$ $1,2,3$ and $j=1,2$, the estimators have the following variances

$$
\operatorname{var}\left(\hat{\theta}_{T_{i}}\right)=\frac{\sigma_{\theta}^{2}}{\left\|\Phi_{i}\right\|^{2}}, \quad \operatorname{var}\left(\hat{\phi}_{T_{j}^{\prime}}\right)=\frac{\sigma_{\phi}^{2}}{\left\|\Phi_{j}^{\prime}\right\|^{2}}
$$


More generally, in the case of any sample time $t_{k}$, the roots are easily computed offline with the Laguerre algorithm [8].

\section{NUMERICAL RESUltS}

Some simulation results are presented below which illustrate the ambiguous trajectories and their estimations. Both the target and the observer have a constant velocity model.

\section{Scenario parameters}

Positions are expressed in meters and velocities in meters/second

- Observer initial state

$$
\begin{aligned}
X_{o}\left(t_{0}\right) & =\left[x_{o}\left(t_{0}\right), \dot{x}_{o}, y_{o}\left(t_{0}\right), \dot{y}_{o}, z_{o}\left(t_{0}\right), \dot{z}_{o}\right]^{T} \\
& =[0,30,0,0,0,5]^{T}
\end{aligned}
$$

- Target initial state

$$
\begin{aligned}
X_{T}\left(t_{0}\right) & =\left[x_{T}\left(t_{0}\right), \dot{x}_{T}, y_{T}\left(t_{0}\right), \dot{y}_{T}, z_{T}\left(t_{0}\right), \dot{z}_{T}\right]^{T} \\
& =[5000,-10,5000,10,100,3]^{T}
\end{aligned}
$$

\section{- Measurements}

Sampling period $=\Delta T=1 \mathrm{~s}$

Number of measurements $=\mathrm{n}=100$

\section{A. Illustration of the ambiguous trajectories}

We present in the following figures the target, the observer, the ambiguous trajectories projected on different planes (Fig. 3-5) and two 3D representation (Fig. 6-7). These trajectories are computed using (8) and set by $\mu \in[0,20]$. They produce the same set of azimuths and elevation angles (here noise-free) (Fig. 2).

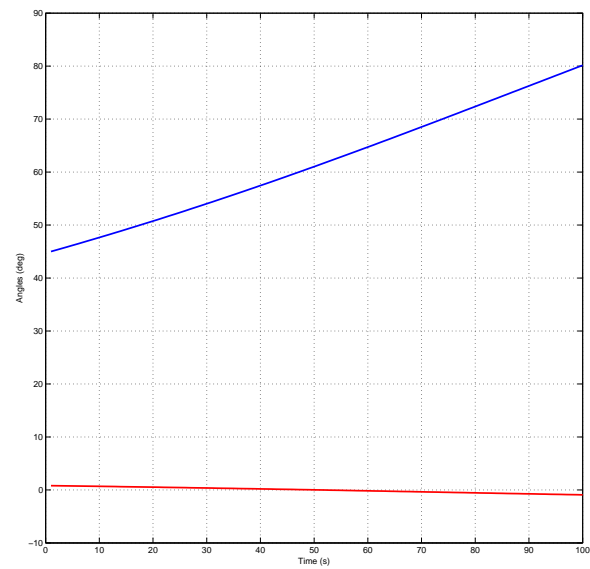

Fig. 2. Azimuths (-) and elevation angles (-)

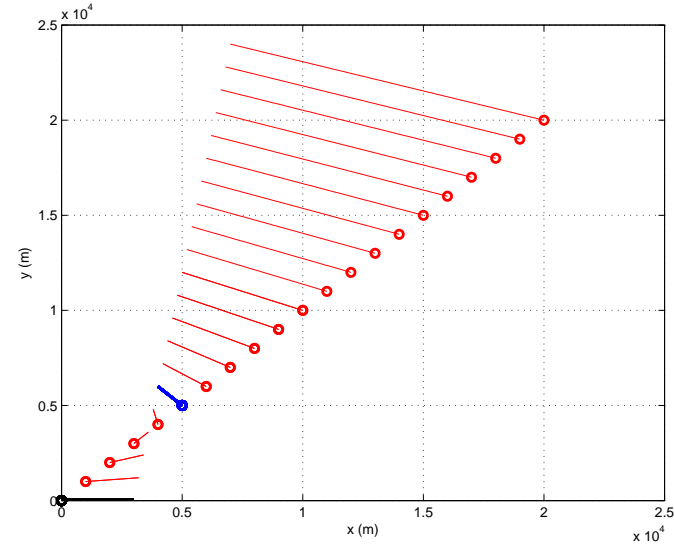

Fig. 3. Ambiguous trajectories. Projection on xy plane. - : ambiguous trajectories. o: initial point. - : target trajectory. - : observer trajectory

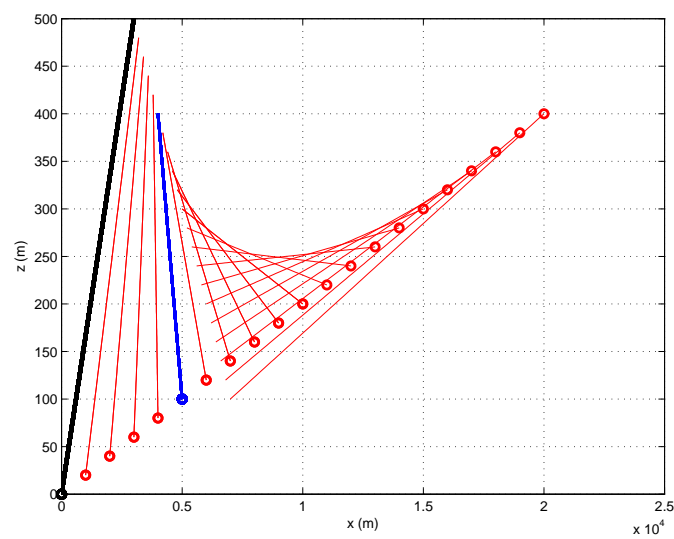

Fig. 4. Ambiguous trajectories. Projection on $\mathrm{xz}$ plane. - : ambiguous trajectories. o: initial point. - : target trajectory. - : observer trajectory

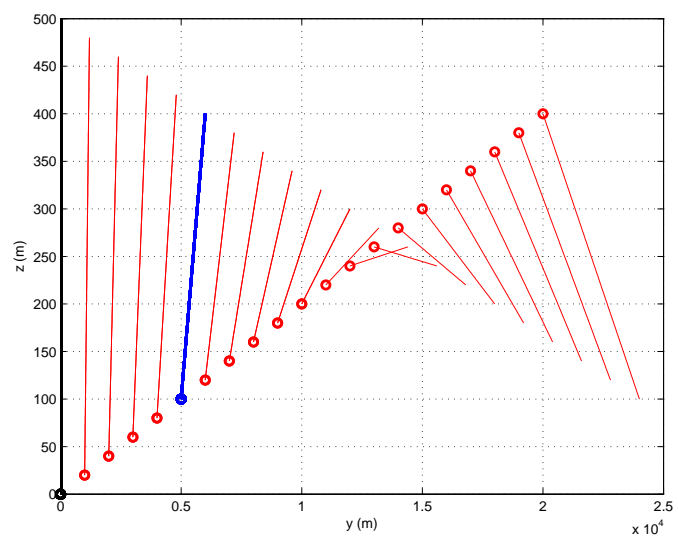

Fig. 5. Ambiguous trajectories. Projection on yz plane. -: ambiguous trajectories. o: initial point. - - target trajectory. - observer trajectory 


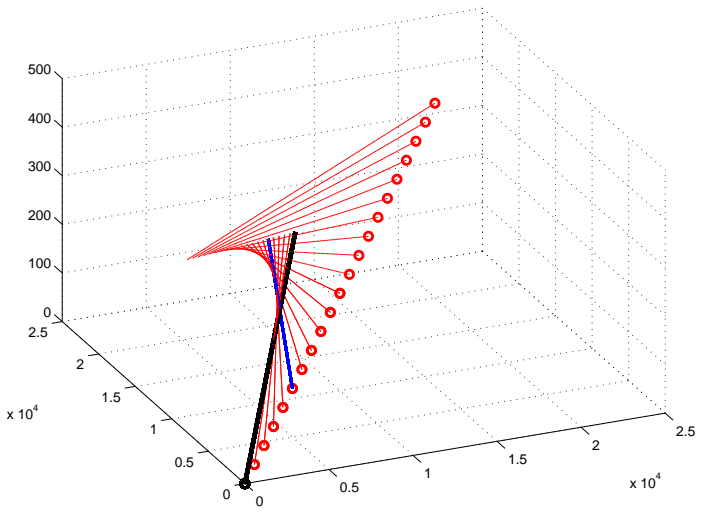

Fig. 6. 3D view of the ambiguous trajectories. - : ambiguous trajectories. o: initial point. - : target trajectory. - : observer trajectory

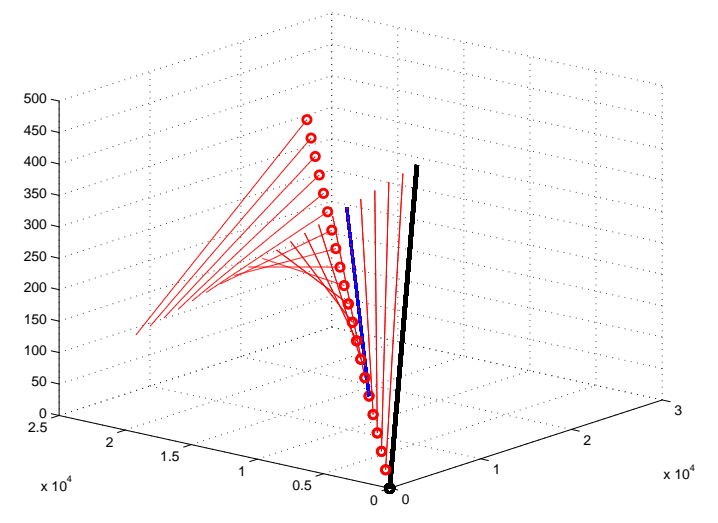

Fig. 7. 3D view of the ambiguous trajectories. - ambiguous trajectories. o: initial point. - : target trajectory. - observer trajectory

We can see on figure 3 that the target's heading $K$ on the xy plane becomes constant as $\mu$ increases. Indeed, we have

$$
\begin{aligned}
& K=\arctan \left(\frac{\dot{x}_{T}}{\dot{y}_{T}}\right)=\arctan \left(\frac{\dot{x}_{o}+\mu \alpha(\Theta)}{\dot{y}_{o}+\mu}\right) \\
& \rightarrow \arctan (\alpha(\Theta)) \text { when } \mu \rightarrow+\infty
\end{aligned}
$$

where $\alpha(\Theta)$ is deduced from (8).

\section{B. Estimation of the ambiguous trajectories}

We present below the performances of the linear estimators (24)-(25). The azimuths and elevation angles are measured every second ( $\Delta T=1 s$ ), thus, we have $n=100$ pairs of angle measurements. The std of the measurements (23) $\left(\sigma=\sigma_{\theta}=\right.$ $\left.\sigma_{\phi}\right)$ are set to $1 \mathrm{deg}$ and $0.1 \mathrm{deg}$. One thousand $(N=1000)$ of Monte Carlo trials have been done in order to estimate the RMSE (root-mean-square error) of the five angles $(\Theta, \Phi)$ :

$$
E_{r}(\hat{\beta}) \triangleq R M S E=\sqrt{\mathbb{E}[\hat{\beta}-\beta]^{2}} \approx \sqrt{\frac{1}{N} \sum_{i=1}^{N}\left[\hat{\beta}^{i}-\beta\right]^{2}}
$$

where $\hat{\beta}^{i}$ is a trial of one the angle estimations $\hat{\theta}_{T_{1}}^{i}, \hat{\theta}_{T_{2}}^{i}, \hat{\theta}_{T_{3}}^{i}, \hat{\phi}_{T^{\prime}}^{i}, \hat{\phi}_{T_{2}^{\prime}}^{i}$. The roots $\left(T_{1}, T_{2}, T_{3}\right)$ and $\left(T_{1}^{\prime}, T_{2}^{\prime}\right)$ are distributed as follows (26)

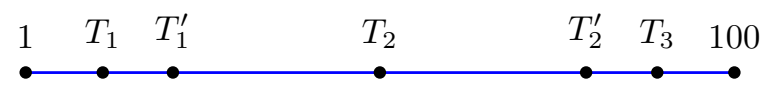

We can see in following table the accuracy of the linear estimators of $(\Theta, \Phi)$. Note that these good performances are valid if the variation amplitude of the angles is not too large The computing time of these estimators is negligible.

TABLE I

$\operatorname{RMSE}$ of $(\hat{\Theta}, \hat{\Phi})(\operatorname{deg})$

\begin{tabular}{|c|c|c|c|c|c|}
\hline$\sigma$ & $E_{r}\left(\hat{\theta}_{T_{1}}\right)$ & $E_{r}\left(\hat{\theta}_{T_{2}}\right)$ & $E_{r}\left(\hat{\theta}_{T_{3}}\right)$ & $E_{r}\left(\hat{\phi}_{T_{1}^{\prime}}\right)$ & $E_{r}\left(\hat{\phi}_{T_{2}^{\prime}}\right)$ \\
\hline $1 \mathrm{deg}$ & 0.18 & 0.15 & 0.19 & 0.14 & 0.14 \\
\hline $0.1 \mathrm{deg}$ & 0.02 & 0.02 & 0.02 & 0.01 & 0.01 \\
\hline
\end{tabular}

We recall that the five angles $(\Theta, \Phi)$ allows us to generate all the ambiguous trajectories by varying the parameter $\mu$ (see proposition 2). Suppose we have an a priori knowledge about some dynamics parameters of the target, like a speed range or a height range. Then it is possible without maneuver to estimate all the trajectories of the target satisfying the constraints using (8) by varying $\mu$. In what follows, we calculate the target trajectories which are compatible with a vertical speed constraint. For example, if the modulus of vertical speed of the target is supposed to be small, precisely $\left|\dot{z}_{T}\right|<0.5 \mathrm{~m} / \mathrm{s}$, than we get the restricted set of trajectories (in green colour) presented in figures (8-11). These trajectories are estimated thanks to the linear estimators (24)-(25) with $\sigma_{\theta}=\sigma_{\phi}=0.1 \operatorname{deg}(23)$.

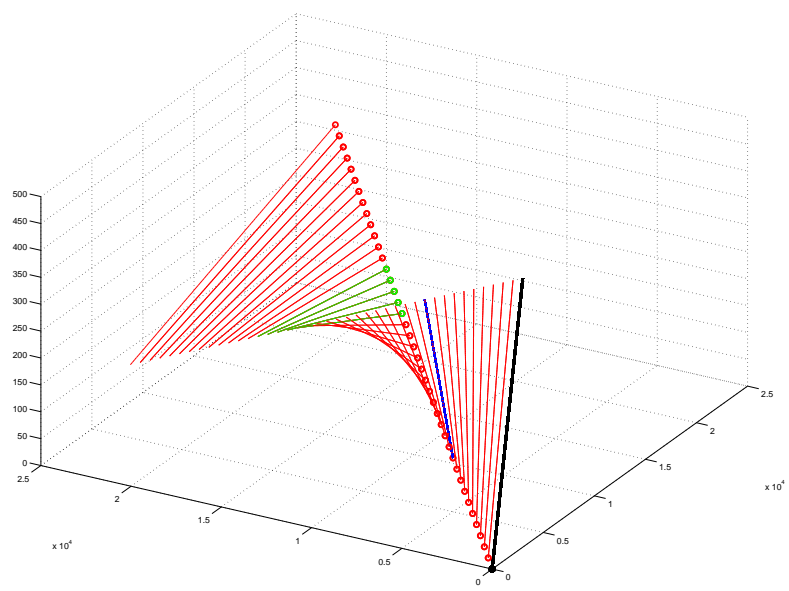

Fig. 8. 3D view of the ambiguous trajectories. -: ambiguous trajectories. o: initial point. -: target trajectory. -: observer trajectory. -: constrained trajectories 


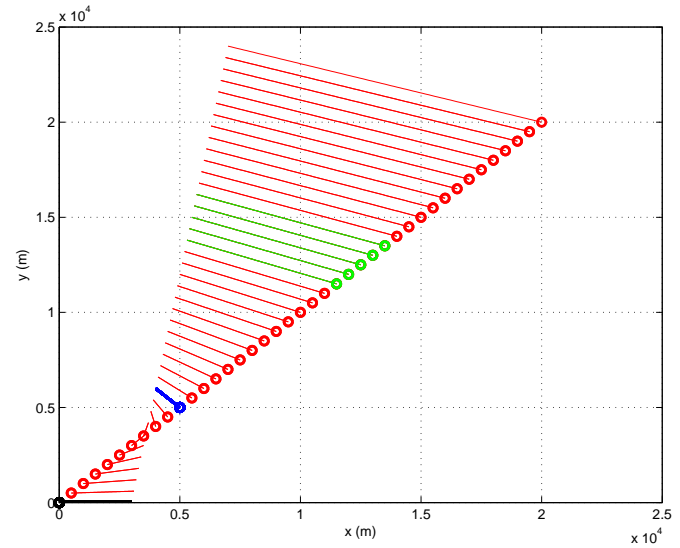

Fig. 9. Ambiguous trajectories. Projection on xy plane. - : ambiguous trajectories. o: initial point. - - target trajectory. : constrained trajectories

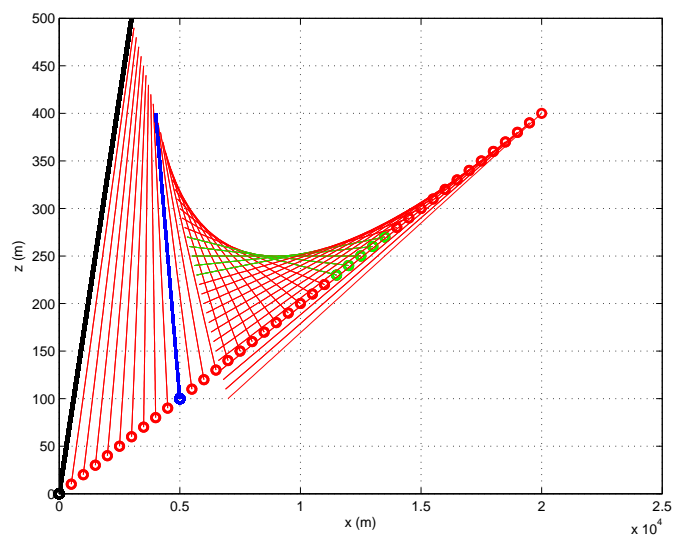

Fig. 10. Ambiguous trajectories. Projection on $\mathrm{xz}$ plane. - : ambiguous trajectories. o: initial point. - : target trajectory. - observer trajectory. : constrained trajectories

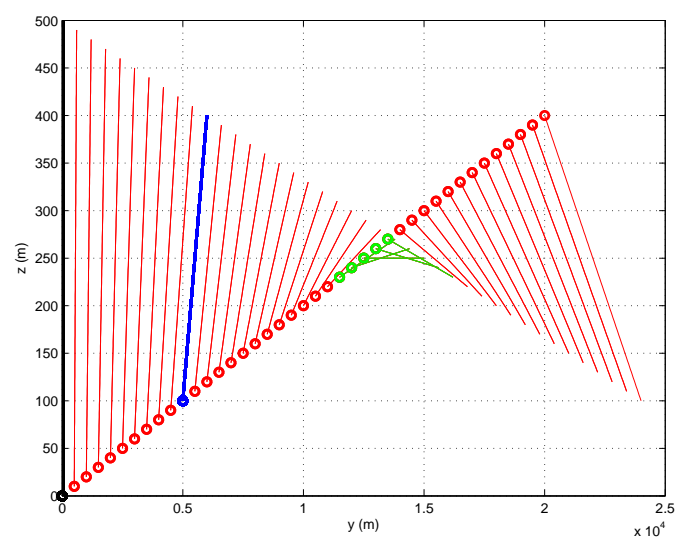

Fig. 11. Ambiguous trajectories. Projection on yz plane. - : ambiguous trajectories. o: initial point. - : target trajectory. - observer trajectory. : constrained trajectories

\section{CONCLUSION}

The paper proposes a method to calculate the set of all the ambiguous target trajectories compatible with the angle measurements in the case of both the observer and the target follow a constant velocity model in a three-dimensional space. These trajectories depend on five observable angles. A quick linear estimator of these angles is presented. Any a priori information about the target state allows us to discriminate among ambiguous trajectories. In doing this, the observer can take a decision to maneuver (or not) in order to estimate accurately the whole 6-dimensional target state.

Acknowledgements. The authors are grateful to Elsa and Juliette for having initiated interesting discussions around this article.

\section{REFERENCES}

[1] Yoko Watanabe, Charles Lesire, Alain Piquereau, Patrick Fabiani, Martial Sanfourche, and Guy Le Besnerais, "The onera ressac unmanned autonomous helicopter: Visual air-to-ground target tracking in an urban environment," in American Helicopter Society 66th Annual Forum (AHS 2010), 2010.

[2] Jean-Michel Passerieux and Dominique Van Cappel, "Optimal observer maneuver for bearings-only tracking," IEEE Transactions on Aerospace and Electronic Systems, vol. 34, no. 3, pp. 777-788, 1998.

[3] J-P Le Cadre and S Laurent-Michel, "Optimizing the receiver maneuvers for bearings-only tracking," Automatica, vol. 35, no. 4, pp. 591-606, 1999.

[4] Jean-Pierre Le Cadre, "Properties of estimability criteria for target motion analysis," IEE Proceedings-Radar, Sonar and Navigation, vol. 145, no. 2, pp. 92-99, 1998.

[5] Claude Jauffret, Denis Pillon, and Annie-Claude Pignol, "Bearings-only tma without observer maneuver," in Information Fusion, 2008 11th International Conference on. IEEE, 2008, pp. 1-8.

[6] Sherry E Hammel and Vincent J Aidala, "Observability requirements for three-dimensional tracking via angle measurements," IEEE Transactions on Aerospace and Electronic Systems, , no. 2, pp. 200-207, 1985.

[7] Eli Fogel and Motti Gavish, "Nth-order dynamics target observability from angle measurements," IEEE Transactions on Aerospace and Electronic Systems, vol. 24, no. 3, pp. 305-308, 1988.

[8] Christian Musso and Nadia Oudjane, "Data reduction for particle filters," in Image and Signal Processing and Analysis, 2005. ISPA 2005. Proceedings of the 4th International Symposium on. IEEE, 2005, pp. 52-57.

[9] Dinh Tuan Pham and Christian Musso, "Une méthode rapide et efficace pour la trajectographie passive par mesures de deux azimuts," in 13 Colloque sur le traitement du signal et des images, FRA, 1991. GRETSI, Groupe d'Études du Traitement du Signal et des Images, 1991.

[10] Christian Musso and Claude Jauffret, "Linear maximum likelihood estimator," in Acoustics, Speech, and Signal Processing, 1991. ICASSP 91., 1991 International Conference on. IEEE, 1991, pp. 1301-1304.

[11] Dinh-Tuan Pham, "Some quick and efficient methods for bearing-only target motion analysis," IEEE transactions on Signal Processing, vol. 41, no. 9, pp. 2737-2751, 1993. 